\title{
BEAM -BEAM STUDY ON THE UPGRADE OF BEIJING ELECTRON POSITRON COLLIDER
}

\author{
Sheng Wang* , Institute of High Energy Physics(IHEP), Beijing, 100049, China \\ Yuanhai Cai, Stanford Linear Accelerator Center (SLAC), Menlo Park, USA
}

\section{Abstract}

It is an important issue to study the beam-beam interaction in the design and performance of such a high luminosity collider as BEPCII, the upgrade of Beijing Electron Positron Collider. The weak-strong simulation is generally used during the design of a collider. For performance a large scale tune scan, the weak-strong simulation studies on beam-beam interaction were done, and the geometry effects were taken into account. The strong-strong simulation studies were done for investigating the luminosity goal and the dependence of the luminosity on the beam parameters.

\section{INTRODUCTION}

BEPCII, a double ring electron positron collider, is an upgrading scheme from Beijing Electron Positron Collider (BEPC) with micro- $\beta$ scheme and multi-bunch collision. To perform multi-bunch collision, two rings of BEPCII will be installed in the existing BEPC tunnel.

To achieve the high luminosity in the factory class collider, high current and small beam size are necessary, and these induce strong beam-beam interaction. The successful performance of KEKB and PEPII indicates that the beam-beam limit can be reached without any single bunch instability. This means the beam-beam interaction limits the luminosity. It is an important issue to study the beam-beam interaction in the design and the performance of such a high luminosity collider.

The beam-beam interaction on BEPCII was studied by simulation. Table 1 shows main parameters of BEPCII, which were used in the simulation. Both weak-strong and strong-strong models were adopted in the simulation. The tune scan was performed for optimizing the tune by weaktrong simulation, while the strong-strong simulation was done for investigating the luminosity goal and the dependence of the luminosity on the beam parameters. The estimation of the parasitic beam-beam effects is also given in this paper.

\section{THE WEAK-STRONG SIMULATION}

The weak-strong simulation studies were done by taking the advantages of the code BBC (Beam-Beam interaction with a Crossing angle) developed by K.Hirata[1]. BBC is a weak strong simulation code in 6dimensional phase space including the effect of crossing angle. Although the weak strong simulation can not investigate the coherent phenomena of beam-beam interaction, it is generally used during the design of a collider.

*E-mail: wangs@ihep.ac.cn
Table 1: Main parameters of BEPCII

\begin{tabular}{||l|c||}
\hline \hline Energy $E(\mathrm{GEV})$ & 1.89 \\
\hline Circumference $C(\mathrm{~m})$ & 237.53 \\
\hline Rev. frequency $f_{0}(\mathrm{MHz})$ & 1.2621 \\
\hline RF frequency $f_{r f}(\mathrm{MHz})$ & 499.8 \\
\hline RF Voltage $V_{r f}(\mathrm{MV})$ & 1.5 \\
\hline Damping time $\tau_{x} / \tau_{y} / \tau_{E}(\mathrm{~ms})$ & $25 / 25 / 12.5$ \\
\hline Total current $/$ beam $I(\mathrm{~A})$ & 0.91 \\
\hline Particle number $N$ & $4.5 \times 10^{12}$ \\
\hline Bunch number $n_{B}$ & 93 \\
\hline Bunch current $I_{b}(\mathrm{~mA})$ & 9.8 \\
\hline Energy spread $\sigma_{\varepsilon}$ & $5.16 \times 10^{-4}$ \\
\hline Momentum compact $\alpha_{p}$ & 0.0235 \\
\hline Bunch length $\sigma_{z}(\mathrm{~cm})$ & 1.5 \\
\hline Tunes $v_{\mathrm{x}} / v_{\mathrm{y}} / \nu_{\mathrm{s}}$ & $6.53 / 7.58 / 0.034$ \\
\hline Emittance $\varepsilon_{x} / \varepsilon_{y}(\mathrm{~nm} \cdot \mathrm{rad})$ & $144 / 2.2$ \\
\hline Beta function at IP $\beta_{x}^{*} / \beta_{y}^{*}(\mathrm{~m})$ & $1 / 0.015$ \\
\hline Beam size at IP $\sigma_{x}^{*} / \sigma_{y}^{*}(\mu \mathrm{m})$ & $380 / 5.7$ \\
\hline Crossing angle $\phi_{c}(\mathrm{mrad})$ & $11 \times 2$ \\
\hline Piwinski angle $\Phi(\mathrm{rad})$ & 0.435 \\
\hline Bunch spacing $s_{b}(\mathrm{~m})$ & 2.4 \\
\hline Beam-beam parameter $\xi_{x} / \xi_{y}$ & $0.04 / 0.04$ \\
\hline \hline
\end{tabular}

The effect of a finite bunch length was taken into account by dividing a strong bunch into 5 slices in longitudinal directions, and the weak bunch is represented by 50 randomly generated super particles, with Gaussian distribution in 6-dimensional phase space. The simulation was done for more than 5 damping time.

The tune scan was performed for optimizing the tune from the view point of high luminosity. Figure 1 shows the simulated luminosity on the tune plane (fractional part only) $\delta v_{x} \in(0,1), \quad \delta v_{y} \in(0,1)$ with crossing angle $\phi_{c}=2 \times 11 \mathrm{mrad}$, in which the luminosity reduction factor $L / L_{0}$ is given instead of luminosity itself. The mesh size was set as 0.02 , which is smaller than the synchrotron tune of $v_{s}=0.034$. It indicates that the high luminosity region is just above the half integer in horizontal plane, and there is no significant deference between just above half integer and just above the integer in vertical plane. According to the commissioning experiences of KEKB and PEPII [2], an above half integer vertical tune is preferable because that the orbit distortion is much stable than that of above an integer. Fig.2 gives a tune scan with 
both transverse tunes above the half integer, with much smaller mesh size. The high luminosity is expected around $\delta v_{x}=0.53$ and $\delta v_{y}=0.58$, and these tune values are set as designed working point.

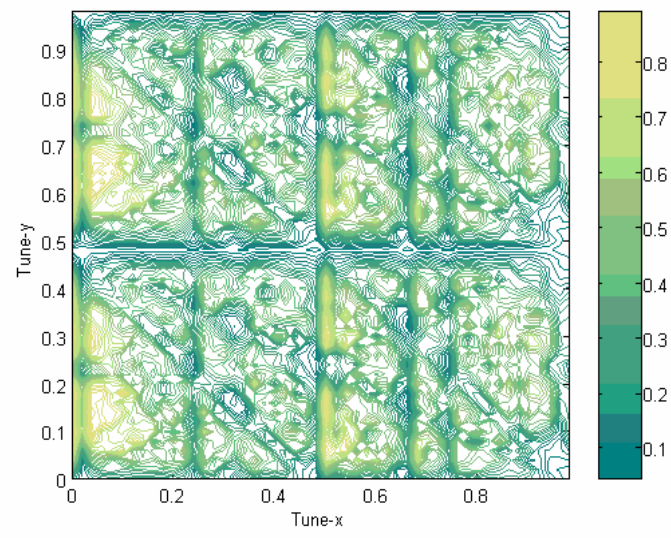

Figure 1: Luminosity survey with crossing angle of $\phi_{c}=11 \times 2 \mathrm{mrad}$

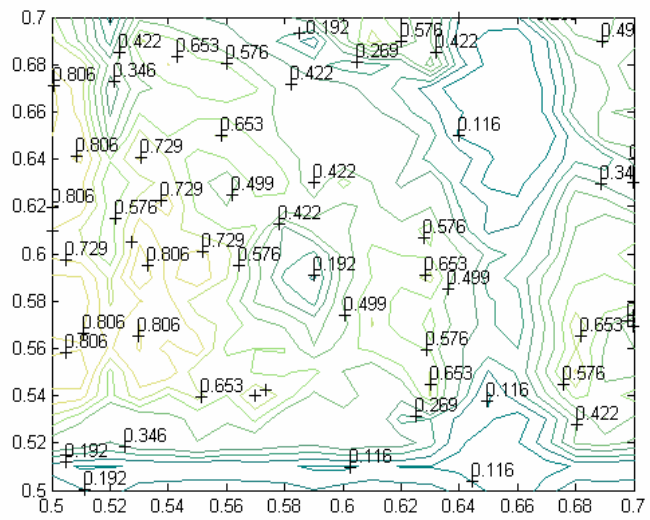

Figure 2: Luminosity survey above the half integer with a crossing angle of $\phi_{c}=11 \times 2 \mathrm{mrad}$

\section{STRONG-STRONG SIMULATION}

\section{The Simulation of Luminosity}

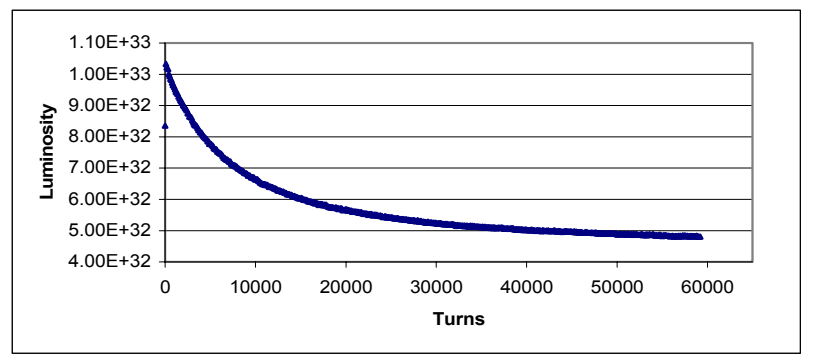

Figure 3: A simulation of luminosity for designed tune with a crossing angle of $\phi_{c}=11 \times 2 \mathrm{mrad}$

The simulation with strong-strong model was done by using Dr. Cai's code[3], which is a 6-D code, including a finite bunch length effect and a crossing angle effect. Fig. 3 shows a simulation result with design tune $\delta v_{x}=0.53$ and $\delta v_{y}=0.58$. After two damping time, beams almost reach their equilibrium.

From the simulation result of Fig.3, there is a large reduction of the luminosity compare with the luminosity goal of $1 * 10^{\wedge} 33$. A local tune scan shows that, much closer of the horizontal tune to half integer, much higher luminosity. This consists with the commissioning experience of KEKB and PEPII. Fig.4 gives a slice of local tune scan, from which, one can find higher luminosity is reached at the tune of $\delta v_{x}=0.51$ and $\delta v_{y}=0.58$, in which the luminosity is $0.62 * 10^{\wedge} 33$. This is chosen as a new tune.

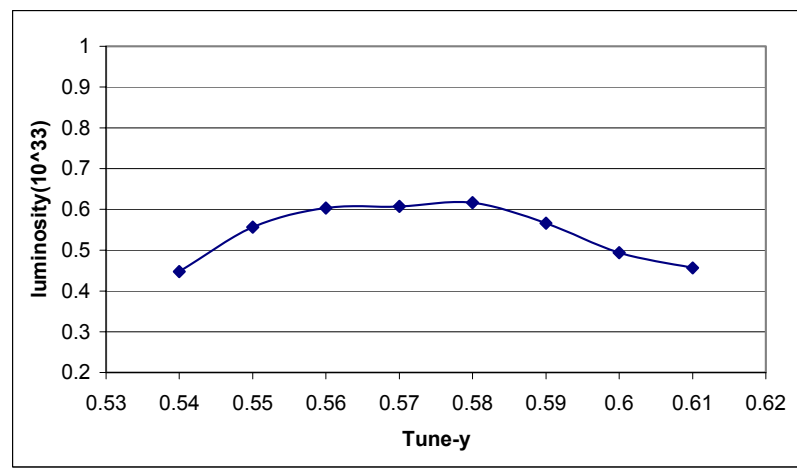

Figure 4: $\delta v_{x}=0.51$, Luminosity vs. $\delta v_{y}$

\section{Beam-Beam Limit}

To achieve higher luminosity, larger beam-beam parameter $\xi_{\mathrm{y}}$ is preferable. However, the maximum $\xi_{\mathrm{y}}$ is limited by beam-beam interaction. Fig. 5 shows the curves of luminosity vs. vertical beam-beam parameter $\xi_{\mathrm{y}}^{2}$. $\xi y=0.04$ is the designed value of BEPCII. It indicates that with half crossing angle of $11 \mathrm{mrad}, \xi_{\mathrm{y}}$ is saturated around 0.06 . From the view point of beam-beam interaction, $\xi_{\mathrm{y}}=0.05$ is reachable. The successful commissioning experience of KEKB and PEPII shows that there is no problem to reach $\xi_{\mathrm{y}}=0.05$.

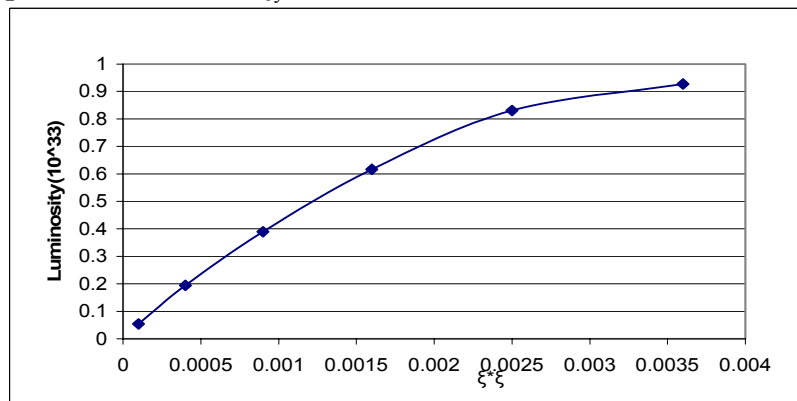

Figure 5: Luminosity vs. $\xi_{\mathrm{y}}^{2}$ with crossing angle of $\phi_{c}=11 \times 2 \mathrm{mrad}$

\section{The Crossing Angle Dependence}

For BEPCII, the crossing angle of $\phi_{c}=11 \times 2 \mathrm{mrad}$ is the basic requirements of interaction region. From the view point of beam-beam interaction, the crossing angle not only limits the maximum $\xi_{\text {y }}$, but also induces some additional luminosity reduction due to the geometric effects. Fig.6 shows that the luminosity reduction factor 
due to finite bunch length effect and crossing angle is about $62 \%$, while the luminosity reduction factor is $87 \%$ in head on scheme. The theoretical geometry effect, which was calculated by using a formula [1] is also given in Fig.6. The luminosity reduction with crossing angle in the strong-strong simulation is much larger than that in the weak-strong simulation.

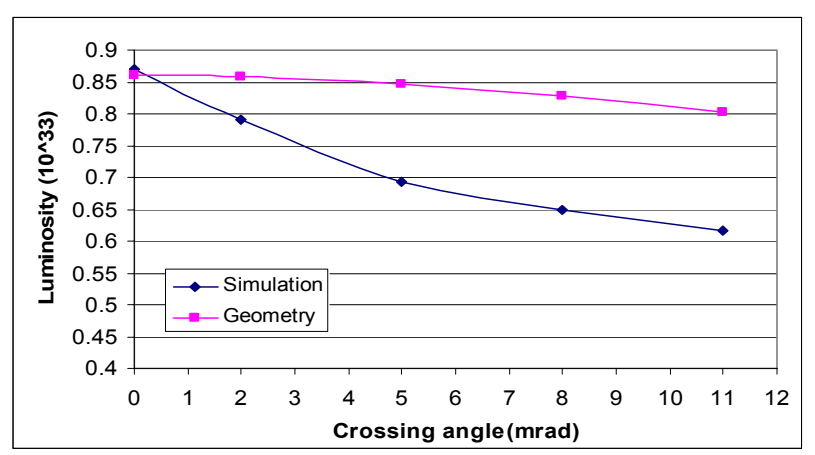

Figure 6: The luminosity as a function of crossing angle. Both the simulation results and theoretical results are given.

\section{The Way to Higher Luminosity}

Due to the finite bunch length effect and crossing angle effect, the simulation luminosity of $0.62 * 10^{\wedge} 33$ is lower than the design goal. The possible ways to increase the luminosity are: increasing the bunch current, decreasing $\beta y$ of IP as well as bunch length, and increasing the bunch number and total beam current, etc. Fig.7 shows

the simulation results with $\xi_{\mathrm{y}}=0.04 / 0.05, \quad \beta_{\mathrm{y}}^{*}=\sigma_{\mathrm{s}}=$ $1.2 \mathrm{~cm} / 1.5 \mathrm{~cm}$ Simulation result shows that when the vertical beam-beam parameter was increased to 0.05 , and $\beta_{\mathrm{y}}^{*}$ and bunch length were decreased to $1.2 \mathrm{~cm}$, the simulation luminosity is more than $1 * 10^{\wedge} 33$. In this case, the total beam current is increased by $25 \%$.

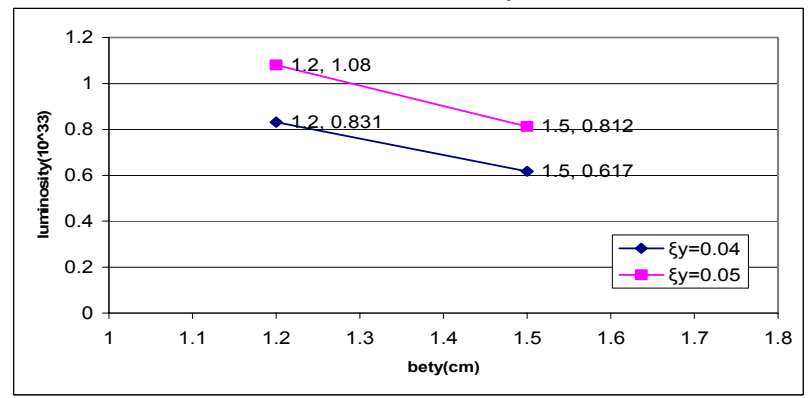

Figure 7: The luminosity vs. $\beta_{\mathrm{y}}^{*} \& \xi_{\mathrm{y}}$

\section{THE PARASITIC BEAM-BEAM EFFECT}

There are 8 parasitic crossings symmetrically located on either side of the IP. The incoherent tune shift $\delta v_{x}$ and $\delta v_{y}$ experienced by a particles at the center of the bunch from a parasitic IP were estimated by Tennyson's formula [4]. Table 2 gives an estimation of the parasitic beambeam effects for different injection pattern, in which, $d$ is the separation between two beams at parasitic IP, and $\Delta s$ is the distance from IP to parasitic IP.

Table 2: The parasitic beam-beam tune shift for different injection pattern

\begin{tabular}{|c|c|c|c|c|c|c|c|}
\hline $\mathrm{E}(\mathrm{GeV})$ & \multicolumn{7}{|c|}{1.89} \\
\hline $\mathrm{I}_{\text {bunch }}(\mathrm{mA})$ & \multicolumn{7}{|c|}{$9.8\left(\mathrm{~N}_{\text {bunch }}=4.84 \mathrm{e} 10\right)$} \\
\hline$\Phi_{\text {crossing }}(\mathrm{mrad})$ & \multicolumn{7}{|c|}{$11 * 2$} \\
\hline $\mathrm{NO}_{\mathrm{pc}}$ & \pm 1 & \pm 2 & \pm 3 & \pm 4 & \pm 5 & \pm 6 & \pm 7 \\
\hline$|\Delta \mathrm{s}|(\mathrm{m})$ & 0.3 & 0.6 & 0.9 & 1.2 & 1.5 & 1.8 & 2.1 \\
\hline $\mathrm{d}(\mathrm{mm})$ & 6.6 & 13.2 & 19.8 & 27.8 & 41.2 & 57.1 & 73.0 \\
\hline$\beta_{\mathrm{x}}$ & 1.09 & 1.36 & 1.81 & 2.71 & 5.26 & 9.53 & 15.08 \\
\hline$\beta_{\mathrm{y}}$ & 6.02 & 24.02 & 54.02 & 88.52 & 88.82 & 79.08 & 69.91 \\
\hline$\Delta v_{x}\left(10^{-3}\right)$ & -0.15 & -0.046 & -0.027 & -0.020 & -0.018 & -0.017 & -0.017 \\
\hline$\Delta v_{\mathrm{y}}\left(10^{-3}\right)$ & 0.81 & 0.81 & 0.81 & 0.67 & 0.31 & 0.14 & 0.077 \\
\hline \multicolumn{2}{|c|}{ Injection patten: By 4} & $\sum \Delta v_{x}\left(10^{-3}\right)$ & \multicolumn{2}{|c|}{-0.04} & $\sum \Delta v_{\mathrm{y}}\left(10^{-3}\right)$ & \multicolumn{2}{|c|}{1.34} \\
\hline \multicolumn{2}{|c|}{ Injection patten: By 3} & $\sum \Delta v_{\mathrm{x}}\left(10^{-3}\right)$ & \multicolumn{2}{|c|}{-0.088} & $\sum \Delta v_{\mathrm{y}}\left(10^{-3}\right)$ & \multicolumn{2}{|c|}{1.90} \\
\hline \multicolumn{2}{|c|}{ Injection patten: By 2} & $\sum \Delta v_{x}\left(10^{-3}\right)$ & \multicolumn{2}{|c|}{-0.166} & $\sum \Delta v_{\mathrm{y}}\left(10^{-3}\right)$ & \multicolumn{2}{|c|}{3.24} \\
\hline \multicolumn{2}{|c|}{ Injection patten: By 1} & $\sum \Delta v_{x}\left(10^{-3}\right)$ & \multicolumn{2}{|c|}{-0.59} & $\sum \Delta v_{\mathrm{y}}\left(10^{-3}\right)$ & \multicolumn{2}{|c|}{7.25} \\
\hline
\end{tabular}

\section{SUMMARY}

The simulation studies with weak-strong and strongstrong models show that: setting the horizontal tune close (above) to half integer is a good choice to get the higher luminosity; the luminosity reduction factor due to finite bunch length effect and crossing angle effect is about $62 \%$, while it is about $87 \%$ in head on scheme; the beambeam parameter of $\xi_{\mathrm{y}}=0.05$ can be reached. The parasitic beam-beam effect was preliminarily studied.

\section{REFERENCES}

[1] K.Hirata, Phys. Rev. Lett. 74(1995)

[2] K.Oide, Operation Experience and Performance Limitations in $\mathrm{e}^{+} \mathrm{e}^{-}$Factories, Proceedings of EPAC 2002, Paris,France

[3] Y.Cai, in PEPII Machine Advisory Committee meeting, 2003.

[4] J.L.Tenneson, Long-Range Forces in APIARY-6, ABC Note 28 (1991). 